\title{
High $Q^{2}$ neutral and charged current interactions in polarized ep collisions at HERA II with the $\mathrm{H} 1$ Detector
}

\author{
Shiraz Habib*, H1 Collaboration \\ DESY \\ E-mail: habib@mail.desy.de
}

Preliminary results from longitudinally polarized electron ${ }^{\dagger}$ collisions with unpolarized protons are presented. The measurements correspond to negative four momentum transfer squared $Q^{2} \gtrsim$ $100 \mathrm{GeV}^{2}$ and are based on the complete HERA-II data set taken by the H1 Detector - 330 $\mathrm{pb}^{-1}$ of data at the centre of mass energy of $\sqrt{s}=318 \mathrm{GeV}$. Measurements demonstrating the parity violating nature of the electroweak force are presented for both neutral current and charged current interactions and are found to be in good agreement with Standard Model predictions. The polarized data sets are combined in order to extract the unpolarized cross section. The resulting single differential $\mathrm{d} \sigma / \mathrm{d} Q^{2}$ and double differential reduced cross sections $\tilde{\sigma}$ (in Bjorken- $x$ and $Q^{2}$ ) are compared to SM expectations for which there is good agreement. The HERA-II unpolarized cross section is combined with its previously published H1 HERA-I measurement to give the HERA-I+II result which has improved statistical as well as systematic precision. The HERA-I+II NC cross sections are used to derive the valence sensitive $x F_{3}^{\gamma Z}$ structure function.

The 2011 Europhysics Conference on High Energy Physics-HEP 2011,

July 21-27, 2011

Grenoble, Rhône-Alpes France

\footnotetext{
${ }^{*}$ Speaker.

†Throughout this text the term "electrons" refers generically to electrons $\left(e^{+}\right)$and positrons $\left(e^{-}\right)$.
} 


\section{Introduction}

The 2000 Luminosity Upgrade of the HERA collider brought with it an increase in specific luminosity by factor 5 and the ability to longitudinally polarize the lepton beam. With electrons at $27.5 \mathrm{GeV}$ and protons at $920 \mathrm{GeV}$, HERA-II provided the laboratory to study deep inelastic scattering with the intent of probing the structure of the proton as well as the chiral structure of the electroweak interaction.

The H1 Detector $[4,5]$ covered the $4 \pi$ solid angle and observed the neutral current (NC: $e p \rightarrow$ $e X)$ and charged current (CC: $\left.e p \rightarrow v_{e} X\right) e^{ \pm} p$ collisions. The reconstruction of both the scattered electron and hadronic final state resulted in overdetermined neutral current kinematics providing a means of optimizing the kinematic reconstruction as well as calibrating the calorimeters.

The measurements presented in this paper[7, 8] are based on the polarized electron data collected by the H1 Detector during the HERA-II run, and amounts to luminosities of $150 \mathrm{pb}^{-1}$ of $e^{-} p$ and $180 \mathrm{pb}^{-1}$ of $e^{+} p$ data. The total, single differential $\mathrm{d} \sigma / \mathrm{d} Q^{2}$ and double differential reduced cross sections $\tilde{\sigma}$ are measured. In the case of neutral current, $\tilde{\sigma}$ is defined by:

$$
\tilde{\sigma}_{N C}^{ \pm}\left(e^{ \pm} p \rightarrow e X\right)\left(x, Q^{2}\right) \equiv \frac{1}{Y_{+}} \frac{x Q^{4}}{2 \pi \alpha^{2}} \frac{d^{2} \sigma_{N C}}{d x d Q^{2}}=\left(Y_{+} \tilde{F}_{2}^{ \pm} \mp Y_{-} x \tilde{F}_{3}^{ \pm}-y^{2} \tilde{F}_{L}^{ \pm}\right) /\left(Y_{+}\right)
$$

where $Y_{ \pm} \equiv 1 \pm(1-y)^{2}$ and $\tilde{F}_{2}, x \tilde{F}_{3}$ and $\tilde{F}_{L}$ are the generalized structure functions. The CC reduced cross section is defined similarly[6]. The reduced cross sections give best sensitivity to the PDFs. Unpolarized HERA-II measurements are made by combining the left- and right-handed samples and correcting for the small residual polarization. The HERA-II unpolarized cross sections are combined with previously published H1 HERA-I measurements using a model independent method[2] which takes into account the correlated systematic uncertainties between the data sets thereby reducing both the statistical and systematic uncertainties.

\section{Results}

Figure 1(a) shows the polarization ${ }^{1}$ dependence of the total charged current cross section compared with its Standard Model (SM) expectation using HERAPDF 1.0[3] $]^{2}$. The data supports the expected linear dependence of the cross section on polarization where the $W$ does not couple to right-handed electrons nor to left-handed positrons. The CC reduced cross sections for the leftand right-handed $e^{+} p$ samples are shown in figure 1(b) for fixed $Q^{2}$ as a function of $x$. Also shown is the SM expectation derived from H1PDF2009[1] which agrees well with the measurement. The combined HERA I+II unpolarized $e^{-} p$ reduced cross section is shown in figure 1(c). The SM expectation together with its up- and down-type quark contributions are also indicated. As can be observed, the measurement provides a strong constraint in determining the up-type parton distribution whose contribution dominates especially at high $x$.

\footnotetext{
${ }^{1}$ The polarization $\mathrm{P}$ of a given sample is defined as the fraction of the sample with right-handed electrons minus the fraction with left-handed electrons, and ranges from -1 to +1 .

${ }^{2}$ Both the HERAPDF 1.0 and H1PDF2009 PDFs are based on measurements using HERA-I data.
} 

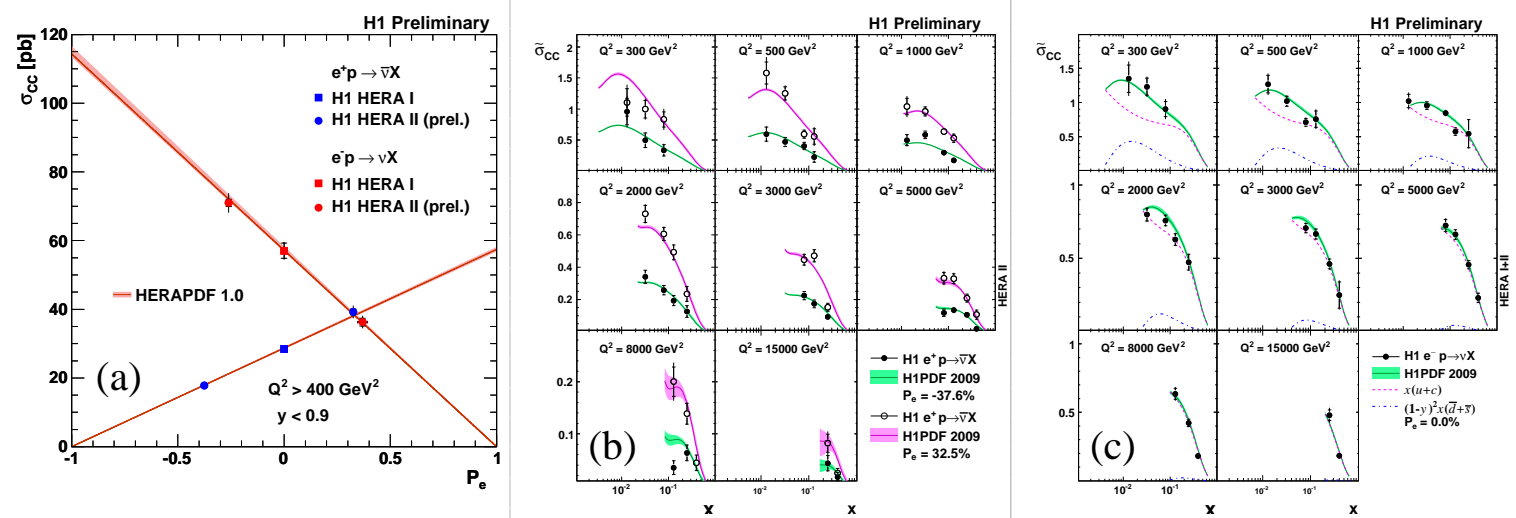

Figure 1: (a) The total CC cross section measurement (points) as a function of polarization against the HERAPDF 1.0 SM expectation (lines). (b) The CC reduced cross section for the left- and right-handed $e^{+} p$ samples. The measurement (points) can be compared to the H1PDF2009 SM expectation (lines). (c) The CC HERA I+II $e^{-} p$ unpolarized reduced cross section. Shown are the measurement (points) and H1PDF2009 SM expectation (full lines) with its quark (dashed lines) and anti-quark (dashed-dotted lines) contributions.

The polarization dependence of the NC cross section is much smaller than that of the CC. It is well quantified by the polarization asymmetry $A$ :

$$
A \equiv \frac{2}{P_{R}-P_{L}} \cdot \frac{\sigma_{R}-\sigma_{L}}{\sigma_{R}+\sigma_{L}}
$$

where $P_{L}$ and $P_{R}$ are the polarization of the left- and right-handed samples respectively and $\sigma_{L}$ and $\sigma_{R}$ the corresponding cross sections. For parity in variance, $A=0$. Figure 2(a) shows $A$ as a function of $Q^{2}$ for $e^{-} p$ and $e^{+} p$ interactions as determined from their respective single differential cross sections. It can be seen that $A$ is non-zero and negative for $e^{-}$, positive for $e^{+}$, and increases in magnitude with $Q^{2}$ as is expected from the SM expectation (also shown) - a confirmation of $\mathrm{NC}$ parity violation.

Figure 2(b) shows the unpolarized HERA-II NC $e^{ \pm} p$ reduced cross sections for fixed $Q^{2}$ as a function of $x$. Good agreement can be observed between the data and the SM prediction (based on the PDFs extracted from HERA-I data). At low $Q^{2}$ the $e^{+}$and $e^{-}$measurements agree. With increasing $Q^{2}$ these measurements begin to separate due to the increasing role of $Z^{0}$ exchange. By first combining the HERA-I and HERA-II unpolarized measurements, the difference between the resulting $e^{-} p$ and $e^{+} p$ HERA-I+II unpolarized cross sections is used to determine $x F_{3}^{\gamma Z}$ as a function of $x$ and $Q^{2}$. Since the dependence on $Q^{2}$ is small, measurements at a fixed $x$-value but different $Q^{2}$ are transformed to a common $Q^{2}$ value of $1500 \mathrm{GeV}^{2}$ and then averaged. Figure 2(d) shows $x F_{3}^{\gamma Z}$ obtained in this way - the sensitivity to the valence quarks is visible from the data which is well described by the SM expectation.

\section{Conclusions}

Parity Violation in both the neutral current and charged current electroweak sectors has been observed and agrees with its Standard Model expectation. The valence sensitive $x F_{3}^{\gamma Z}$ structure function has been determined and is well described by the Standard Model. 


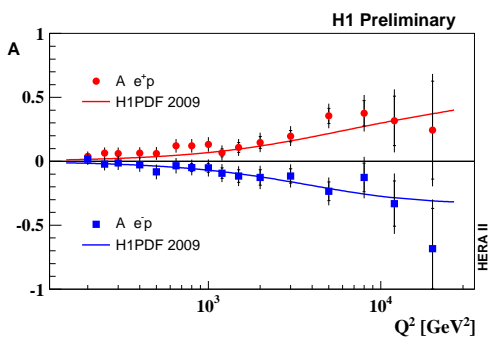

(a)

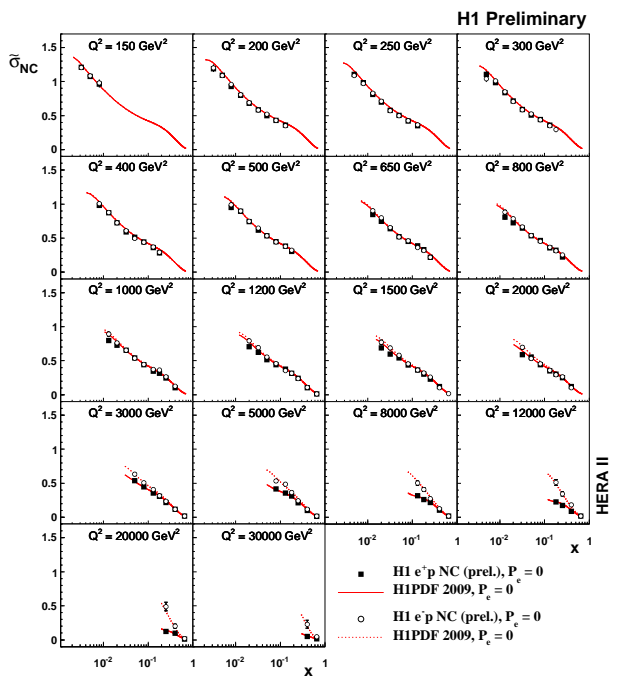

(b)

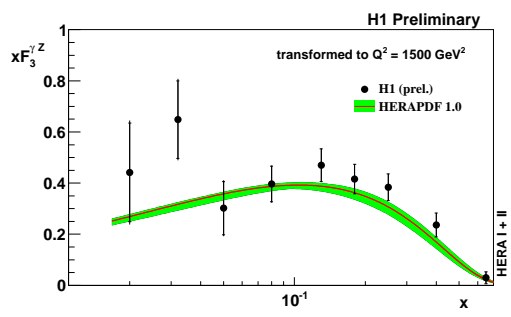

(c)

Figure 2: (a) The NC polarization asymmetry $\mathrm{A}$ for $e^{-} p$ (blue squares) and $e^{+} p$ (red circles) data with its H1PDF 2009 SM expectation ( $e^{-} p$ as blue and $e^{+} p$ as red lines). (b) The unpolarized HERA-II NC reduced cross sections for $e^{+} p$ (solid squares) and $e^{-} p$ (open circles) interactions. Also shown are the corresponding H1PDF $2009 \mathrm{SM}$ expectations ( $e^{+} p$ as solid and $e^{-} p$ as dashed lines). (c) The $x F_{3}^{\gamma Z}$ structure function as a function of $x$ for $Q^{2}=1500 \mathrm{GeV}^{2}$. The measurement (points) is shown together with its HERAPDF 1.0 SM expectation (line).

The neutral and charged current inclusive cross sections have been measured and agree well with their Standard Model expectations. These measurements provide strong constraints for determining the parton distributions of the proton and will therefore play a significant role in establishing our view of the proton and making predictions at the LHC.

\section{References}

[1] F. D. Aaron et al. A Precision Measurement of the Inclusive ep Scattering Cross Section at HERA. Eur. Phys. J., C64:561-587, 2009.

[2] F. D. Aaron et al. Measurement of the Inclusive ep Scattering Cross Section at Low Q2 and $x$ at HERA. Eur.Phys.J., C63:625-678, 2009.

[3] F. D. Aaron et al. Combined Measurement and QCD Analysis of the Inclusive ep Scattering Cross Sections at HERA. JHEP, 01:109, 2010.

[4] I. Abt et al. The H1 detector at HERA. Nucl.Instrum.Meth., A386:310-347, 1997.

[5] I. Abt et al. The Tracking, calorimeter and muon detectors of the H1 experiment at HERA. Nucl.Instrum.Meth., A386:348-396, 1997.

[6] C. Adloff et al. Measurement and QCD analysis of neutral and charged current cross sections at HERA. Eur. Phys. J., C30:1-32, 2003.

[7] H1 Collaboration. High Q2 Charged Current in polarised ep collisions at HERA II. H1prelim-09-043, 2009.

[8] H1 Collaboration. High Q2 Neutral Current in polarised ep collisions at HERA II. H1prelim-09-042, 2009. 